problems associated with the differential equation

$$
\frac{\partial^{2} u}{\partial x^{2}}+\frac{\partial^{2} u}{\partial y^{2}}+\lambda u=0
$$

for various types of boundary conditions when the boundary is rectangular.

Purdue University

\title{
ON THE CONVERGENCE OF A CONTINUED FRACTION
}

\section{T. F. GLASS AND WALTER LEIGHTON}

It is known [1] that sufficient conditions for the convergence of the continued fraction

$$
b_{0}+\frac{a_{1}}{1}+\frac{a_{2}}{1}+\cdots
$$

where the elements are complex numbers, are

$$
\left|a_{2}\right| \geqq 5, \quad\left|a_{2 n}\right| \geqq 25 / 4, \quad\left|a_{2 n-1}\right| \leqq 1 / 4, \quad n=2,3,4, \cdots .
$$

The purpose of this note is to extend this result.

THEOREM. If $\left|a_{2 n+1}\right| \leqq r \leqq 1 / 4(n=1,2,3, \cdots)$ and if the numbers $a_{2 n}=\rho_{2 n} e^{i \theta_{2 n}}(n=1,2,3, \cdots)$ satisfy the conditions

$$
\begin{array}{lrl}
\rho_{2 n} & \geqq 2(1+r)^{2}\left[1-\cos \left(\theta_{2 n}+\theta_{0}\right)\right], & 0 \leqq \theta_{2 n}<\pi-\theta_{0}, \\
\rho_{2 n} \geqq 4(1+r)^{2}, & \pi-\theta_{0} \leqq \theta_{2 n} \leqq \pi+\theta_{0}, \\
\rho_{2 n} \geqq 2(1+r)^{2}\left[1-\cos \left(\theta_{2 n}-\theta_{0}\right)\right], & \pi+\theta_{0}<\theta_{2 n} \leqq 2 \pi,
\end{array}
$$

where $\theta_{0}=2$ arc $\sin r$, the continued fraction (1) converges.

To prove the theorem we employ the continued fraction

$$
1+\frac{x_{1}}{1}+\frac{x_{2}}{1}+\cdots
$$

where

$$
x_{2 n}=\frac{\left(1+a_{2 n-1}\right)\left(1+a_{2 n+1}\right)}{a_{2 n}}, \quad n=2,3, \cdots
$$

Presented to the Society, September 5, 1941; received by the editors May 7, 1942. 


$$
\begin{aligned}
x_{2 n+1} & =a_{2 n+1}, \\
x_{1} & =-a_{1}, \quad x_{2}=\frac{1+a_{3}}{a_{2}} .
\end{aligned}
$$$$
n=1,2, \cdots,
$$

Since the convergence of (1) is independent of the choice of $b_{0}$, we set $b_{0}=1-a_{1}$, and it follows [1] that the $2 n$th convergent of (1) formally equals the $(2 n+1)$ st convergent of $(6)$, while the $(2 n+1)$ st convergent of (1) is equal to the $2 n$th convergent of (6). Thus (1) and (6) will converge or diverge together. By the first hypothesis of the theorem and (7.2) the numbers $x_{2 n+1}$ are bounded and lie in $\bar{R}$ the closed parabola $|z|-R(z)=1 / 2$. It is thus sufficient to prove that the numbers $x_{2 n}$ defined in (7.1) and (7.3) subject to conditions (3), (4), and (5) are bounded and lie in the parabolic region described above [2].

To this end let

$$
\begin{aligned}
s_{2 n+1} & =\left(1+a_{2 n-1}\right)\left(1+a_{2 n+1}\right)=a_{2 n} x_{2 n} \\
& =r_{2 n+1} e^{i \phi_{2 n+1}}, \quad n=2,3, \cdots,
\end{aligned}
$$

and set $x_{2 n}=t_{2 n} e^{i \omega_{2 n}}$. It is clear that $t_{2 n} \leqq \max r_{2 n+1} / \min \rho_{2 n}=$ $(1+r)^{2} / \min \rho_{2 n}$.

First suppose that $a_{2 n}$ lies in the region defined by (4). Then $\min \rho_{2 n}=4(1+r)^{2}, \quad t_{2 n} \leqq 1 / 4$ and $x_{2 n}$ will lie in $\bar{R}$. Next suppose that (3) holds, that is, that $a_{2 n}$ lies outside the cardioid $\rho=2(1+r)^{2}\left[1-\cos \left(\theta+\theta_{0}\right)\right]$ and in the angle $0 \leqq \theta<\pi-\theta_{0}$. Hence $\min \rho_{2 n}=2(1+r)^{2}\left[1-\cos \left(\theta+\theta_{0}\right)\right]$ for each $\theta$ and thus

$$
t_{2 n} \leqq \frac{1}{2\left[1-\cos \left(\theta_{2 n}+\theta_{0}\right)\right]}, \quad 0 \leqq \theta_{2 n}<\pi-\theta_{0} .
$$

Further $\omega_{2 n}=\phi_{2 n+1}-\theta_{2 n}$ from which it follows that $-\theta_{0}-\theta_{2 n} \leqq \omega_{2 n}$ $\leqq \theta_{0}-\theta_{2 n}$, and hence that $-\pi \leqq \omega_{2 n} \leqq \theta_{0}$. It is clear that the right-hand member of (8) decreases steadily from $1 / 2\left(1-\cos \theta_{0}\right)$ to $1 / 4$ as $\theta_{2 n}$ increases from 0 to $\pi-\theta_{0}$ and at the same time $\omega_{2 n}$ decreases steadily from $\phi_{2 n+1}$ to $\phi_{2 n+1}+\theta_{0}-\pi$. The proof for the case when (3) holds may now be completed by proving that the point $\left(1 / 2\left(1-\cos \theta_{0}\right), \theta_{0}\right)$ and the points

$$
\left(1 / 2\left[1-\cos \left(\theta+\theta_{0}\right)\right],-\theta-\theta_{0}\right), \quad 0 \leqq \theta<\pi-\theta_{0},
$$

lie in $\bar{R}$, as an examination of a simple figure will show. The first point evidently lies on the parabola since the equation of the parabola in polar coordinates is

$$
r=\frac{1}{2(1-\cos \theta)}
$$


The substitution $\lambda=-\theta-\theta_{0}$ proves immediately that the points (9) lie on (10).

By symmetry it is clear that when $a_{2 n}$ lies in the region defined by (5) the corresponding complex number $x_{2 n}$ lies in $\bar{R}$. This completes the proof of the theorem.

Corollary 1. If $\left|a_{2 n+1}\right| \leqq r \leqq 1 / 4$ and $\left|a_{2 n}\right| \geqq 4(1+r)^{2}$, the continued fraction (1) converges.

It is clear that an analogous theorem to the above may be proved with the roles of the even and the odd elements interchanged.

\section{BIBLIOGRAPHY}

1. Walter Leighton and H. S. Wall, On the transformation and convergence of continued fractions, Amer. J. Math. vol. 58 (1936) pp. 267-281.

2. W. T. Scott and H. S. Wall, A convergence theorem for continued fractions, Trans. Amer. Math. Soc. vol. 47 (1940) pp. 155-172.

The Marion Institute and

The Rice Institute 